\title{
Estratégias de Enfrentamento do Estresse Desenvolvidas por Estudantes de Medicina da Universidade Federal de Santa Catarina*
}

\author{
Stress Coping Strategies Developed by \\ Medical Students of the Federal University \\ of Santa Catarina
}

PALAVRAS-CHAVE:

- Currículo;

- Educação médica;

- Qualidade de vida;

- Estudantes de Medicina;

- Estresse.

KEY-WORDS:

- Curriculum;

- Education, Medical;

-Quality of life;

- Students, Medical;

- Stress.

Recebido em: 14/08/2006

Aprovado em: 26/09/2006
Ronaldo Zonta ${ }^{1}$ Ana Carolina Couto Robles ${ }^{2}$ Suely Grosseman ${ }^{3}$

\section{RESUMO}

Em um estudo qualitativo sobre a qualidade de vida de estudantes de Medicina, na Universidade Federal de Santa Catarina, emergiu o tema "Estratégias de enfrentamento do estresse". O objetivo deste artigo é apresentar dados relativos a este tema, na perspectiva de melhorar a qualidade de vida do estudante de Medicina. Seguindo princípios da pesquisa qualitativa, o tema proposto é apresentado com base na análise dos dados empíricos, relativos a depoimentos selecionados do conjunto de dados dos 25 participantes do estudo e no diálogo com a literatura. Entre os que desenvolveram estratégias, as apontadas incluíam valorização dos relacionamentos interpessoais e de fenômenos do cotidiano, equilíbrio entre estudo e lazer, organização do tempo, cuidados com a saúde, alimentação e o sono, prática de atividade física, religiosidade, trabalhar a própria personalidade para lidar com situações adversas e procura por assistência psicológica. Sugere-se maior preocupação dos envolvidos na educação médica com a qualidade de vida do estudante de Medicina e o desenvolvimento de estratégias para promovê-la ou que preparem o estudante para lidar com o estresse durante a formação médica.

\begin{abstract}
The theme "Stress coping strategies"came up in the course of a qualitative study about the quality of life of medical students at the Federal University of Santa Catarina. The objective of this article is to present data related to this issue in the hope of improving the quality of life of the medical student. According to the principles of qualitative research, the theme proposed is presented based on the analysis of empirical data derived from selected statements of the 25 particpants of the study and from the literature. Those participants who developed strategies mentioned among others the importance of interpersonal relationships and day-to-day phenomena, balance between study and leisure, organization of one's time, physical exercise, health nutrition and sleep, religiosity, working the own personality to deal with adverse situations and seek for psychological assistance. One recommends a greater concern of those involved in medical education with the quality of life of the students and the development of strategies to promote this behavior or to prepare the students to cope with the stress during medical education
\end{abstract}

\footnotetext{
* Trabalho vencedor do prêmio ABEM/2006 de Educação Médica no 44ํㅡ Congresso Brasileiro de Educação Médica, no período de 21 a 24 de setembro de 2006, Gramado Rio Grande do Sul, Brasil.

${ }^{1}$ Medicina da Família, Hospital Conceição, Porto Alegre, Brasil.

${ }^{2}$ Universidade Federal de Santa Catarina, Porto Alegre, Brasil.

${ }^{3}$ Universidade Federal de Santa Catarina, Porto Alegre, Brasil.
} 


\section{INTRODUÇÃO}

A preocupação com a qualidade de vida do estudante de Medicina vem sendo alvo de estudos em diversos países. Pesquisas demonstram a presença de fatores estressantes na educação médica e sua conseqüência para a saúde dos estudantes $^{1-4}$

Enns et al ${ }^{5}$ enfatizam que fatores estressantes - como pressão para aprender, grande quantidade de novas informações, falta de tempo para atividades sociais e contato com doenças graves e com a morte no cuidado clínico dos pacientes - podem contribuir para o aparecimento de sintomas depressivos nos estudantes. E Costa e Pereira ${ }^{6}$ discorrem sobre os diversos tipos de abuso vivenciados pelos estudantes de Medicina, que podem agravar seu estresse.

Como decorrência, estudos têm demonstrado alta prevalência de suicídio, depressão, uso de drogas, distúrbios conjugais e disfunções profissionais em médicos e estudantes de Medicina, que podem prejudicar o cuidado do paciente ${ }^{7-11}$.

Alguns autores enfatizam que a redução do estresse e a melhoria da qualidade de vida do estudante seriam uma forma de promover neles a criatividade e a iniciativa, capacitando-os a lidar melhor com as necessidades psicossociais dos pacientes 12,13 . Como ressalta Jung14: "somente se o médico souber lidar com ele mesmo e com seus próprios problemas ele será capaz de ensinar o paciente a fazer o mesmo".

No primeiro semestre de 2003, o novo currículo do curso de Medicina da Universidade Federal de Santa Catarina (UFSC) foi implantado, tendo como uma de suas metas a boa qualidade de vida do estudante.

Para avaliar esta meta, foi realizado, no segundo semestre de 2004, o estudo intitulado "A percepção de estudantes que vivenciam o novo currículo do curso de medicina da UFSC sobre sua qualidade de vida" e apresentado em 200515. O método utilizado foi o qualitativo, tipo estudo de caso, sendo a amostra selecionada aleatoriamente por sorteio e composta por 25 estudantes, do universo de 49 estudantes que estavam cursando, na época, a quarta fase do curso. O projeto da pesquisa foi aprovado pelo Comitê de Ética em Pesquisas com Seres Humanos da UFSC, em junho de 2004, sob o protocolo de número 197/2004. A coleta de dados foi realizada por meio de entrevista semi-estruturada em profundidade ${ }^{16,17}$, seguindo todos os preceitos éticos.

Um dos temas emergentes foi "Estratégias individuais e coletivas para lidar com o estresse". Entendendo que este tema é de grande importância, o objetivo deste artigo é apresentar dados relativos a ele, na perspectiva de melhorar a qualidade de vida do estudante de Medicina. Partindo dessas considerações e seguindo princípios da pesquisa qualitativa, o tema proposto é apresentado a partir de dados empíricos, relativos a depoimentos selecionados do conjunto de dados dos participantes do estudo e analisados com apoio da literatura.

\section{ESTRATÉGIAS INDIVIDUAIS E COLETIVAS PARA LIDAR COM O ESTRESSE}

Dos 25 entrevistados, 13 eram do sexo feminino e 12 do masculino.

Ao refletirem sobre as estratégias utilizadas para promover sua qualidade de vida, alguns mencionaram ter características pessoais que os protegem do estresse:

Eu sou um cara mais relaxado, não sou tão preocupado com as pressões.

Eu sempre tive esta questão da consciência tranqüila. Nunca procurei ir dormir sem estar com as coisas resolvidas.

Outros comentaram que, como sua atitude diante da vida sempre foi administrar o tempo de forma a conciliar o estudo com as outras dimensões de sua vida, eles conseguiam lidar bem com o curso:

(...) quando eu posso, tento dedicar um tempo pra mim.

Eu não deixo de fazer as coisas que eu gosto.

Entretanto, muitos referiram ter que se esforçar para mudar de postura frente ao estresse enfrentado e para separar a dimensão do estudo das outras dimensões da vida.

Foi decisão de vida, que eu não vou chorar mais, que eu não vou me estressar (...). Eu resolvi, porque não vale a pena (...). Foi uma procura minha, de começar a refletir (...) comecei a pensar na vida, nunca tinha feito isso (...). Tenho só 20 anos! Daí, imagina quando eu tiver 40, eu vou estar uma velha caquética, né? (...) Eu decidi que eu não queria ficar mais desse jeito, que se tivesse ruim, não importa, não ia me estressar, eu ia fazer de um jeito que fosse ser legal (...) buscar outras coisas. O principal que me ajudou a estar de bem comigo. (...) Aprendi a me cobrar um pouco menos, também.

(...) quando pisava em casa, tentava esquecer os problemas, esquecer tudo... Pensava: "Agora vou ficar com a minha mãe, vou pedalar, vou nadar... Vou aprender a separar as coisas, senão a minha vida vai virar um inferno. Eu vou acabar com a minha saúde...". Não foi fácil porque são muitos problemas juntos, muita ansiedade misturada com angústia. (... Tive que fazer uma mudança interna, (...) acho que melhorei bastante (...) porque eu aprendi a dividir as coisas. A buscar um equilíbrio. (...) 
Falta muito pra aprender. Mas, essa questão de saber separar as coisas, buscar alguma coisa pra suprir é bem importante. (...) Tenho dificuldade ainda de administrar a minha angústia, a minha ansiedade. Vencer os obstáculos. Mas eu já pude crescer com isso.

Não me estresso mais tanto com os problemas do currículo atual (...). Eu acho que o fator principal foi parar de me estressar com as coisas que eu não poderia mudar. Teve uma época que eu simplesmente parei com tudo e agora eu estou vendo quais as lutas que eu posso ter e quais as coisas que não me adianta lutar, que eu não vou conseguir mudar. (...) Onde que eu tenho que cuidar da minha vida e onde que eu posso lutar para melhorar o currículo, que é o que eu acho que todo mundo pensa em relação ao curso. Então, têm coisas que eu parei de me estressar e eu acho que isso foi o principal motivo para eu melhorar a minha qualidade de vida.

Eu vou pra praia às vezes. Durante a semana, eu saio, dou uma volta (...). Tento estudar bastante durante a semana pra no fim de semana ficar mais tranqüila.

$\mathrm{Eu}$ acabo conseguindo relativamente manter uma qualidade de vida por conseguir separar as coisas. Manter-me bem, feliz fazendo os dois: (...) me esforçar durante a semana no lado profissional, no caso, estudo, e no fim de semana ter um lado bem pessoal.

(...) ter algumas prioridades (...) Medicina é minha prioridade, mas tenho outras (...) eu gosto de artes, fazer alguma coisa com artes.

Neste sentido, um dos entrevistados ressaltou que o principal fator que fez melhorar sua qualidade de vida foi "trabalhar em cima da (sua própria) personalidade, ser uma pessoa mais animada".

Alguns buscaram reforçar as relações interpessoais com família, amigos, namorados e/ou novos círculos de amizade no meio extra-universitário:

... comecei a conversar mais com meus pais (...) o relacionamento em casa melhorou. (...) Isso já me fez ficar um pouco melhor.

Sair com o namorado, ver os amigos (...). Tento fazer coisas pequenas, mas que me deixam feliz.

Estou saindo mais, conhecendo mais gente.

(...) procurei expandir minha vida fora da Medicina, fazendo curso de línguas, conversando com gente diferente.

A valorização do cotidiano da vida e da natureza também foi mencionada:
Vou ver um pôr-do-sol na Lagoa. Isso me faz feliz. Eu gosto das coisas pequenas. Às vezes, a gente viaja no fim de semana, pra sair da rotina. Mas, durante a semana, essas coisas pequenas já me deixam feliz.

(...) eu estou procurando me animar com coisas bobas (...) tentando ver o lado bom das coisas, pra me animar (...) tentando me animar com coisas cotidianas.

Para uma entrevistada, o engajamento em trabalho como professora propiciou maior percepção de seu próprio valor:

Outra coisa que me ajudou muito a melhorar, (...) a me sentir melhor, foi que eu comecei a trabalhar no ano passado, comecei a dar aula particular. Com isso, quando eu comecei a ajudar as pessoas, passar aquilo que eu sabia, (...) eu comecei a me sentir mais útil, (...) me ajudou bastante. (...) Mesmo com pouco tempo, eu ia na boa. Meus alunos ligavam dizendo que tinham tirado dez na prova. Aí eu me realizava todo dia: "Se eu faço isso bem... posso então estudar e fazer uma coisa legal um dia com o paciente!". Foi uma coisa que me animou bastante.

O resgate de hábitos de vida saudáveis, de cuidados com a saúde, de lazer e de cursos que haviam sido abandonados também foi citado:

Eu estou nadando, pedalando. Pude voltar pro meu inglês. (...) Eu corro na beira-mar.

O lazer é uma coisa que eu prezo muito, e às vezes até deixo umas responsabilidades de lado pro lazer, pra não ficar doido. (...) Alimentação saudável... (...) Prezo muito as horas de sono, (...) exercício físico... (...) Tento ter momentos de lazer nos finais de semana.

Às vezes, eu tento ficar em casa fazendo qualquer coisa, vendo TV, ouvindo música. (...) Tentar tirar um pouco a cabeça disso, (...) estou tentando cuidar da minha saúde.

A religiosidade também foi uma estratégia comentada: "eu comecei a ir mais à Igreja, a rezar...".

A organização do tempo para estudo e de um ambiente propício para este foi mencionada:

(...) nível de organização (...) cada dia estudar um pouco.

(...) estudo a hora que eu quero. Tenho um lugar pra estudar. É bom ter um horário para estudar, pra se concentrar. Estudar a hora que der vontade.

Uns estão buscando uma mudança de atitude, tentando primeiro se conhecer melhor, por meio de assistência psicológica: "Eu também faço consulta com um psicólogo, então venho podendo me entender". 
Mas alguns ainda não conseguiram desenvolver estratégias: Já comecei a fazer atividade física, já parei. Eu já pensei em iniciar um hobby, mas não tirei do papel.

Ultimamente, não consigo fazer nada para promover a minha qualidade de vida.

Ultimamente, não tenho feito muita coisa. (...) Eu deveria estar fazendo exercícios físicos, mas não estou, porque me sinto cansada a maior parte do tempo. Eu não consigo. E eu não tenho feito mais nada.

O não desenvolvimento de estratégias para lidar com o estresse tem acarretado graves conseqüências sobre a saúde destes entrevistados:

Eu não tenho vontade de estudar. Uma vez, eu gostava de estudar, tipo eu não tenho aquela vontade de viver, de fazer certas coisas. Na verdade, eu não tenho vontade de fazer nada, pois eu estou bastante cansada da Medicina. Não da Medicina em si. Eu entrei na faculdade, sabia que ia ser isso, cansativo e tal... mas tem sido muito mais do que eu esperava.

Minha saúde não é tão boa quanto já foi. Eu ando com muita dificuldade para dormir, insônia mesmo. Estou com gastrite.

Entre as estratégias apontadas estão a valorização dos relacionamentos interpessoais com parentes, namorados, amigos e colegas, a busca de maior equilíbrio entre estudo e lazer, bem como reserva de tempo para praticar atividade física, cuidar da alimentação, dormir e cuidar da saúde.

Foi enfatizada ainda a questão de trabalhar com a própria personalidade, buscando sentir-se feliz e ter ânimo, evitando sentir-se estressado ou pressionado com as situações desfavoráveis.

Enquanto certos estudantes mencionaram que conseguem lidar naturalmente com as situações de estresse presentes no processo de ser estudante de Medicina, alguns tiveram que procurar assistência profissional de um psicólogo, ao passo que outros não estão conseguindo lidar com o estresse a que estão submetidos, o que influencia sua saúde.

Os que lidam bem com situações de estresse referiram que isto decorria de características pessoais que lhes permitiam se manter bem mesmo quando a maioria considerava sua qualidade de vida prejudicada.

Algumas estratégias apontadas pelos entrevistados também foram encontradas por outros estudiosos. Lee e Graham² observaram entre as estratégias de redução do estresse mais freqüentemente desenvolvidas por estudantes de Medicina: compartilhar experiências relacionadas ao estresse com ou- tras pessoas (o que alguns referiram como "estar no mesmo barco"), manter boas relações interpessoais (família, amigos, namorado/a), tirar "folgas" da Medicina (descansar por alguns momentos em casa), praticar atividade física e ter religiosidade. Além disto, alguns estudantes utilizavam técnicas de relaxamento, meditavam e/ou freqüentavam concertos musicais.

Em estudo qualitativo com 130 médicos, as práticas que estes utilizavam para promover seu bem-estar também incluíam a valorização dos relacionamentos interpessoais (passar mais tempo com a família e os amigos), ter atividades religiosas ou espirituais e cuidar de si mesmo, além de achar um sentido no trabalho, estabelecer limites e adotar uma visão filosófica positiva, tal como ser positivo ou se concentrar no sucesso $^{18}$.

Vários pesquisadores têm tentado identificar o que faz com que alguns estudantes de Medicina lidem de uma forma mais ou menos traumática com as situações estressantes do seu dia-a-dia ${ }^{5,19-21}$. Wolf ${ }^{20}$ enfatiza a importância de identificar os estudantes de maior risco de desajustamentos psicológicos durante seu treinamento.

Miller e Surtees ${ }^{21}$, estudando a evolução de sintomas de ansiedade e depressão entre estudantes de Medicina do primeiro ano em seus primeiros seis meses na universidade, concluíram que um pequeno subgrupo continuamente sintomático continha estudantes mais lentos para fazer amizades, com suporte inapropriado dos familiares, com tendência de brigar, com namorados(as) firmes e/ou personalidades "vulneráveis". Por outro lado, o subgrupo que esteve bem por todo o período tinha experimentado uma infância com muitos cuidados, raramente tinha namorados(as), mostrou pequena tendência para brigas e tinha personalidade "adaptativa".

Stewart et al22, em estudo com 140 estudantes de Medicina, observaram que os que apresentaram menor sucesso no desempenho acadêmico possuíam elevados níveis de sintomas e de ideações depressivas. Estudantes com traços de ansiedade tinham maior susceptibilidade ao estresse, enquanto o oposto se dava com os mais otimistas e/ou com estilos ativos de lidar com o estresse (confronto com as situações, esforço para alterá-las).

O perfeccionismo tem sido apontado como um traço da personalidade freqüente em estudantes de Medicina e tem forte relação com estresse psicológico ${ }^{23}$. Em estudo realizado com a população em geral, Blatt ${ }^{24}$ demonstrou forte associação entre perfeccionismo e aumento de riscos de depressão, ansiedade, sintomas obsessivo-compulsivos e suicídio.

Thayler, Newman e McClaim ${ }^{25}$ estudaram, sistematicamente, estratégias para regular o humor. Os autores identifi- 
caram, categorizaram e avaliaram a efetividade de comportamentos geralmente usados para regular o humor. Dentre todas as categorias, o método mais efetivo foi o exercício físico. Em ordem decrescente, foram descritos os seguintes itens: escutar música, interação social, diferentes tipos de técnicas cognitivas (controlar pensamentos, fazer um discurso de incentivo próprio ou pensar positivo) e praticar outras atividades, como hobbies, atividades recreacionais, assistir televisão, escrever, ler ou trabalhar.

Os meios menos efetivos para diminuir o mau humor foram ficar sozinho e evitar a pessoa ou coisa que o causava. Para reduzir a ansiedade, a tensão e o nervosismo, as estratégias mais efetivas foram a prática de exercícios físicos e de relaxamento. Os autores concluem que a maneira mais efetiva de combater o mau humor seria o "manejo ativo do humor", combinando exercícios, relaxamento e técnicas cognitivas. As menos efetivas seriam o "manejo passivo do humor" (que inclui assistir TV, comer e dormir) e a "redução direta da tensão" (uso de drogas ou atividade sexual). Para diminuir a tensão, os autores sugerem a busca de atividades prazerosas e para aumentar a energia, as técnicas cognitivas e a prática de atividade física e social.

Kersley $^{26}$ menciona alguns conselhos práticos para que médicos possam manter ou recuperar seu bem-estar. Entre eles estão a valorização do lado pessoal, a busca por pessoas que dêem suporte emocional, a busca de equilíbrio entre mente, corpo e espírito, o desenvolvimento do otimismo e a valorização do que se faz e do próprio potencial.

Como se notou, alguns estudantes conseguiram lidar bem com as situações de estresse do processo de ser estudante de Medicina por possuírem características que lhes permitem manter uma boa qualidade de vida, independentemente dos fatores estressantes a que são submetidos. Em outro extremo, alguns nem mesmo conseguiram desenvolver uma estratégia para lidar com estes fatores.

Por isso, é necessário criar espaços para que todo estudante de Medicina possa refletir sobre as angústias vivenciadas no seu dia-a-dia e discuti-las. Também é importante que as escolas médicas tenham como prover suporte psicológico e pedagógico aos estudantes que não conseguem lidar adequadamente com essas situações.

Cox et al27 ponderam que todos os esforços devem ser feitos para elevar a saúde global e o bem-estar dos estudantes de Medicina, e para incrementar o número de programas de promoção à saúde a eles direcionados. Como afirma Wolf ${ }^{20}$, estudantes de Medicina saudáveis provavelmente serão médicos saudáveis, tornando-se modelos de estilos de vida saudáveis para seus pacientes.
Para Rospenda, Halpert e Richman ${ }^{28}$, o uso de estratégias de gerenciamento do tempo pode ser particularmente útil na redução do estresse em estudantes de Medicina. Os autores sugerem que talvez seja apropriado que as escolas médicas criem estratégias de gerenciamento do tempo.

Shapiro, Shapiro e Schwartz ${ }^{29}$, ao fazerem revisão da literatura, afirmam que programas de manejo do estresse na escola médica - como o ensino de técnicas para lidar com o estresse (meditação, hipnose, relaxamento muscular) e a promoção de grupos de suporte para expressão das emoções e da afeição - podem ser muito positivos para seus participantes. A partir dos estudos, eles comentam que estudantes de Medicina que participam de programas de manejo do estresse apresentam aumento da função imunológica, diminuição de sintomas de depressão e ansiedade, aumento da espiritualidade e da empatia, elevação do conhecimento sobre terapias alternativas para referenciá-las futuramente, aumento do conhecimento sobre os efeitos do estresse e maior uso de habilidades positivas para lidar com ele.

Os autores concluem que a maioria das intervenções desenhadas para reduzir o estresse na educação médica se mostrou efetiva e que praticamente todos os participantes consideraram os programas úteis. Mas ponderam que ainda faltam estudos que demonstrem o tipo de programa mais efetivo e a duração e freqüência necessárias para que possa surtir efeitos.

Uma proposta que tem sido utilizada por diversas faculdades de Medicina é o programa de tutoria. Bellodi et al ${ }^{30}$ mencionam que "o desenrolar de todo o processo de formação, especialmente em relação às escolhas a realizar, pode ser muito facilitado se for oferecido ao aluno contato com modelos identificatórios adequados e estimulantes para as suas diferentes necessidades". Para esses autores, o objetivo da atividade tutorial é o suporte pessoal durante o desenvolvimento profissional. O programa de tutoria também cria oportunidades de identificar problemas durante a formação e seus possíveis encaminhamentos visando a soluções.

Para Nogueira-Martins ${ }^{31}$, é "fundamental a criação de serviços de orientação psicopedagógica para os estudantes". Seguindo essa idéia, várias escolas médicas do Brasil vêm organizando setores de apoio aos estudantes, estruturados de acordo com as peculiaridades institucionais de cada escola, denominados Núcleos ou Centros de Apoio Psicopedagógicos ${ }^{32,33}$.

Nogueira-Martins ${ }^{31}$ também reflete sobre a importância de se incluir a dimensão psicológica.na formação do estudante de Medicina Para o autor, "o trabalho de sensibilização do jovem aluno em relação aos seus aspectos psicológicos - motivações para a profissão, idealização do papel do médico, etc. 
- e às suas reações vivenciais durante o curso de Medicina é uma medida de atenção primária". Isto poderia ser concretizado mediante modificações curriculares nas escolas médicas que incluam o ensino de psicologia médica, centrado nas vicissitudes do curso médico e do exercício da Medicina, "propiciando ao estudante um espaço para entrar em contato com seus sentimentos e emoções, diante dos seres humanos que está começando a atender. Um espaço que priorize a reflexão e a troca de experiências".

Nesse sentido, Grosseman e Patrício ${ }^{12,34}$ sugerem que se inclua e valorize a dimensão psicológica e o cuidado no currículo, no aprendizado teórico-prático, no suporte aos estudantes e, fundamentalmente, nas relações vivenciadas, no cotidiano, entre profissionais envolvidos com a educação e os discentes, entre discentes e discentes, e entre estes e todos os que participam de seus espaços de aprendizagem (entre eles, profissionais de saúde e indivíduos da comunidade/paciente).

\section{CONSIDERAÇÕES FINAIS}

Diversos caminhos podem ser utilizados para promover a qualidade de vida do estudante de Medicina. Cabe também aos educadores da área da saúde a responsabilidade do cuidado no ensino e na prática da relação médico-paciente/comunidade, da relação profissional e da relação educador-educando, pois as atitudes do cotidiano valem mais que as palavras. A confirmação vivenciada da teoria é a mais potente fonte de aprendizado.

A escola médica deve estar preparada e ter em foco esta preocupação, cuidando mais do estudante de Medicina, respeitando-o, escutando-o, ajudando-o a desenvolver estratégias que o preparem para lidar com a pressão que vivenciará no cotidiano acadêmico e profissional, bem como dando suporte psicológico e pedagógico de forma geral e em especial àqueles que passam por dificuldades.

\section{REFERÊNCIAS}

1. Villanueva T, Haivas I. Studying medicine and quality of life. Student BMJ 2006;14:170-1.

2. Lee J, Graham AV. Students' perception of medical school stress and their evaluation of a wellness elective. Med Educ. 2001; 35(7): 652-9.

3. Millan LR, Souza EM, De Marco OLN, Rossi E. Assistência psicológica ao estudante de medicina. In: Marcondes, E.; Gonçalves, E. L, orgs. Educação médica. São Paulo: Savier; 1998. p. 340-354.

4. Supe AN. A study of stress in medical students at Seth G.S. Medical College. Journal of Postgraduate Medicine. 1998; 44(1): 1-6.
5. Enns MW, Cox BJ, Sareen J, Freeman P. Adaptive and maladaptive perfectionism in medical students: a longitudinal investigation. Med Educ. 2001; 35(11):1034-42.

6. Costa LSM, Pereira CAA. O abuso como causa evitável de estresse entre estudantes de medicina. Rev Bras Educ Méd. 2005; 29(3):185-190.

7. Ritson B. Alcohol and medical students. Med Educ. 2001; 35(7):622-623.

8. Webb E, Ashton CH, Kelly P, Kamah F. An update on British medical students' lifestyles. Med Educ. 1998; 32(3):325-331.

9. Richings JC, Khara GS, Mcdowell M. Suicide in young doctors. British Journal of Psychiatry. 1986; 149:475-8. [SAI]

10. Meleiro A. Suicídio entre médicos e estudantes de medicina. Rev Ass Med Bras 1998; 44(2):135-140.

11. Rossetto MAC, Skawinski LSR, Coelho ACP, Rossetto JAJ, Bolla K. Avaliação das características psicológicas dos estudantes de medicina por meio do método de rorschach. Psikê - R. Curso Psicol Cent Univ. FMU. São Paulo. 2000 jul-dez; 5(2):41-51

12. Grosseman S. Satisfação com o trabalho: do desejo à realidade de ser médico [Tese]. Florianópolis: UFSC; 2001.

13. Grosseman S, Patrício ZM. Do deseja à realidade de ser médico: a educação e a prática médica como um processo contínuo de construção individual e coletiva. Florianópolis: Editora da UFSC;2004. 203 p.

14. Jung CG. Memórias, sonhos e reflexões. Rio de Janeiro: Nova Fronteira;1975.

15. Zonta R. A percepção de estudantes que vivenciam o novo currículo do curso de medicina da UFSC sobre sua qualidade de vida. [Monografia] Florianópolis: Universidade Federal de Santa Catarina; 2005.

16. Bogdan RC, Biklen SK. Investigação qualitativa em educação. Portugal: Porto Editora;1994.

17. Minayo, MC de S. O desafio do conhecimento: pesquisa qualitativa em saúde. São Paulo: Editora Hucitec;2004.

18. Weiner EL, Swain GR, Wolf B, Gottlieb M. A qualitative study of physicians' own wellness-promotion practices. West J. Med. 2001; 174(1):19-23.

19. Stewart SM, Betson C, Lam TH, Marshall IB, Lee PWH, Wong CM. Predicting stress in first year medical students: a longitudinal study. Med Educ. 1997; 31(3):163-8.

20. Wolf TM. Stress, coping and health: enhancing well-being during medical school. Med Educ. 1994; 28(1):8-17.

21. Miller PM, Surtees PG. Psychological symptoms and their course in first-year medical students as assessed by the 
Interval General Health Questionnaire (I-GHQ). Br J Psychiatry 1991; 159:199-207.

22. Stewart SM, Betson C, Marshall I, Wong CM, Lee PW, Lam TH. Stress and vulnerability in medical students. Med Educ. 1995; 29(2):119-27.

23. Henning K, Ey S, Shaw D. Perfectionism, the impostor phenomenon and psychological adjustment in medical, dental, nursing and pharmacy students. Med Educ. 1998; 32(5):456-464.

24. Blatt S. The destructiveness of perfectionism: implications for the treatment of depression. American Psychologist 1995; 50:1003-20.

25. Thayer RE, Newman JR, McClaim TM. Self-regulation of mood: strategies for changing a bad mood, raising energy, and reducing tension. J Pers Soc Psychol. 1994; 67(5):910925.

26. Kersley SE. Wellbeing: reality or dream? [journal article]. BMJ. 2003; 326 (sup 109) Disponível em: http://bmj.com/ cgi/content/full/326/7391/S109?ct.

27. Cox C, Cambre KM, Wolf TM, Webster MG, Hooper J. Trends in the number and administrative characteristics of medical school health promotion programmes. Med Educ. 2001; 35(2):173-4.

28. Rospenda KM, Halpert J, Richman JA. Effects of social support on medical students' performances [Journal Article]. Academic Medicine. 1994; 69(6):496-500.

29. Shapiro SL, Shapiro DE, Schwartz GE. Stress management in medical education: a review of the literature. Academic Medicine. 2000; 75(7):748-59.
30. Bellodi PL, Martinho T, Massaroppe B, Martins MA, Santos MAS. Temas para um programa de tutoria em medicina: uma investigação das necessidades dos alunos da FMUP. Rev Bra de Educ Méd. 2004; 28(2):119-127.

31. Nogueira-Martins LA. Saúde mental dos profissionais de saúde. In: Botega, H. J, org. Prática Psiquiátrica no hospital geral: interconsulta e emergência. Porto Alegre: Artmed Editora; 2002. p. 130-144.

32. Gonçalves EL. Apoio psicológico e pedagógico aos estudantes de medicina. Boletim informativo Ponto e Vírgula do centro de desenvolvimento da educação médica da FMUSP 1994; 30:8-12.

33. Ramos-Cerqueira, A. T. A. et al. Once upon a time... fairy tales and psychodrama to help students at the end of the medical course. Interface - Comunic., Saúde, Educ.,2004/ 2005; 9 (16):81-89.

34. Grosseman S, Patrício ZM. A relação médico-paciente e o cuidado humano: subsídios para promoção da educação médica. Rev Bras Educ Méd. 2004 ; 28(2):99-105.

\section{Conflito de Interesse}

Declarou não haver.

\section{Endereço para correspondência}

Ronaldo Zonta

Rua Duarte Schütel,215/1002/ Bl.A Centro

88015-640 Florianópolis- SC

E-mail: rzonta@terra.com.br 